\title{
Determination of the charge transfer resistance of poly $(3,4-$ ethylenedioxythiophene)-modified electrodes immediately after overoxidation
}

\author{
D. Zalka ${ }^{a}$, N. Kovács ${ }^{a}$, K. Szekeres ${ }^{a}$, M. Ujvári ${ }^{a}$, S. Vesztergom ${ }^{a}$, S. Eliseeva ${ }^{b}$, V. \\ Kondratiev $^{b}$, G.G. Láng ${ }^{a, *}$ \\ ${ }^{a}$ Institute of Chemistry, Laboratory of Electrochemistry and Electroanalytical Chemistry, Eötvös Loránd \\ University, Pázmány P. s. 1/A, H-1117 Budapest, Hungary \\ ${ }^{b}$ Chemical Department, St.Petersburg State University, Universitetskii pr. 26, 198504, Russia
}

\begin{abstract}
Many electrochemical systems are intrinsically nonstationary and are affected by timedependent phenomena. The requirement of stationarity in the classical version of impedance spectroscopy appears to be in conflict with the essential properties of the object, therefore a post-experimental mathematical/analytical procedure is necessary for the reconstruction of the "true" impedance values. In this study, it has been shown that the 4-dimensional analysis method, originally proposed by Stoynov, can not only be used for the correction of existing (experimentally measured) impedance data, but it also opens up the possibility of the estimation of impedance spectra outside the time interval of the measurements. As an illustrative example the method has been applied for the determination of the charge transfer resistance $\left(R_{\mathrm{ct}}\right)$ of poly(3,4-ethylenedioxytiophene) (PEDOT) modified electrode as a function of time, including the $R_{\mathrm{ct}}$ value corresponding to the time instant just after overoxidation of the polymer film. After the overoxidation the charge transfer resistance decreased continuously with experiment time to a value somewhat higher than the $R_{\mathrm{ct}}$ of the pristine electrode. The results imply that a "healing process" may occur at the film/substrate interface. A better understanding of this effect may have an impact on practical applications.
\end{abstract}

Keywords: non-stationary system; overoxidation of poly(3,4-ethylenedioxytiophene) (PEDOT); 4-dimensional analysis method; instantaneous impedance; charge transfer resistance;

\footnotetext{
${ }^{*}$ Corresponding author at: Institute of Chemistry, Department of Physical Chemistry, Laboratory of Electrochemistry and Electroanalytical Chemistry, Eötvös Loránd University, Pázmány P. s. 1/A, H-1117 Budapest, Hungary

E-mail: langgyg@chem.elte.hu
}

This paper is dedicated to Professor Zdravko Stoynov on the occasion of his 80th birthday in recognition of his great contribution to electrochemistry. 


\section{Introduction}

There are several impedance measurement methods used in electrochemistry which mainly differ in the type of the excitation signal (e.g. sinusoidal, multi-sinusoidal, white noise, etc.). Nevertheless, the single sine excitation method is by far the most popular. When this method is employed the system under investigation is sequentially excited by applying small sinusoidal waves of a quantity, such as current or voltage. This is done within a given frequency range (e.g. from some $\mathrm{mHz}$ to some $\mathrm{MHz}$ ). The response of the system is an alternating voltage or current signal with the same frequency as that of the input signal. The frequency dependence of the response can be attributed to specific processes occurring either at the interfaces or inside the phases in contact.

The impedance at a given frequency is the complex ratio of the Fourier transforms of the voltage and the current signals (sinusoids of the same frequency) for a two-terminal circuit, which fulfill the criteria of stationarity, linearity and causality under investigated conditions. A frequency spectrum can be obtained by sweeping the excitation frequency. Unfortunately, single sine impedance spectroscopy measurements suffer from increasing time consumption if the frequency range is extended toward lower frequencies. When data recording occurs at low frequencies a complete measurement sequence can take significant time (at least several minutes). However, many electrochemical systems are intrinsically nonstationary and are affected by time-dependent phenomena. So, the requirement of stationarity in the classical version of impedance spectroscopy appears to be in conflict with the essential properties of the object. Strictly speaking, in such cases the obtained results are not "impedances" and cannot be used directly in any analysis.

According to Stoynov [1] there are two general cases in the impedance measurements related to the non-stationary errors:

(i) measurements in a stationary system under non-steady state conditions;

(ii) investigation of non-stationary systems.

The error of the first type increases sharply with decrease of the frequency (see ref. [2]). In the second case, an additional error appears, related to the measurement delay due to the classical "frequency by frequency" mode of impedance measurements.

Different methods to deal with a non-stationary behavior can be found in literature. Stoynov proposed a method of determining instantaneous impedance diagrams for nonstationary systems based on a four-dimensional approach $[1,3]$. (The instantaneous impedance is defined as an instantaneous projection of the non-stationary state of the system into the 
frequency domain [1].) Darowicki et al. developed a dynamic EIS method to trace the dynamics of the degradation process by the calculation of an instantaneous impedance [4]. In [5] a procedure was proposed to quantify and correct for the time-evolution by means of the calculation of an instantaneous impedance.

The method of Stoynov (the 4-dimensional analysis) provides for correction of the systematic errors, arising during the measurements of time-evolving impedance, i.e. when the consecutive impedance measurements are performed at different system states, but each of the measured impedance values can be accepted as "valid" in the classical sense. Of course, in this case the individual values (especially those measured at the lower frequencies) may be still corrupted by typical errors caused by the system evolution during the experiment [6]. (If the problem is related to the mathematical basis of the transfer function analysis [2,7], the so called rotating Fourier transform can be used to overcome this limitation [6,8].)

As discussed before, the four-dimensional analysis method is based on the assumption for the continuum of the object's state and parameters space. It requires a number of impedance spectra recorded subsequently at the same set of frequencies. Every measured data at a given frequency should additionally contain the time of measurement. Thus, the experimental data form a set of 4-dimensional arrays, containing frequency, real and imaginary components of the impedance and the time of measurement. The post-experimental analytical procedure previews the reconstruction of calculated instantaneous impedances. For every measured frequency two one dimensional functions of "iso-frequency dependencies" (e.g. for the real and for the imaginary components or for the magnitude and phase angle of the complex immittance) are constructed. Then, each iso-frequency dependence is modeled by an approximating formal model. On the basis of the continuity of the evolution, interpolation (and/or extrapolation) is performed resulting in instantaneous projections of the full impedance-time space and "reconstructed" instantaneous impedances related to a selected instant of the time (e.g. the beginning of each frequency scan, but the procedure can be applied to any time instants). Thus a set of impedance diagrams is obtained, containing "instantaneous" (virtually simultaneously recorded) impedances. Each of these diagrams can be regarded as a stationary one, free of nonsteady-state errors. On the basis of the above, one could conclude that the four-dimensional analysis method can be most effective in the correction of low-frequency impedance data. However, as it will be shown in the present study, this technique can also be used for solving other problems.

For instance, it is known that the impedance spectra of overoxidized poly(3,4ethylenedioxytiophene) (PEDOT) films on gold recorded in aqueous sulphuric acid solutions 
differ from those measured for freshly prepared films [9]. The most interesting feature is the appearance of an arc (or a "depressed semicircle") at high frequencies in the complex plane impedance plot. The published results support the mechanistic picture, according to which the originally compact and strongly adherent polymer films undergo structural changes during the overoxidation (degradation) process [10-15]. Overoxidation may result in the partial delamination of the polymer layer and may lead to the exposure of some parts of the underlying metal substrate to the electrolyte solution [12]. It should be emphasized here that the polymer film still present on the substrate after (partial) overoxidation remains electroactive, and its internal structure may be an interesting subject for further studies, since according to literature reports conducting polymers in different overoxidation states show unique features useful for analytical, sensing and biomedical applications [16-20].

The time evolution of the impedance spectra is another remarkable feature of the electrodes with overoxidized PEDOT films. To our knowledge, this phenomenon was first reported in ref. [21]. According to this observation, the impedance spectra change continuously over several hours when the electrode potential is held in the "stability region" after overoxidation of the film. This means that the impedance spectra recorded using the consecutive frequency sweep mode (typical of EIS) are corrupted by typical errors caused by the system evolution during the experiment. On the other hand, for reasons of measuring technology, we are not able to record an impedance spectrum immediately after the overoxidation process. But even if we would be able to do so it wouldn't solve the problems related to the nonstationarity of the system. Nevertheless, it is beyond doubt that the knowledge of the "initial" impedance parameters like charge transfer resistances, high and low frequency capacitances, etc., may be essential for a better understanding of the degradation/overoxidation process and it may also have an impact on practical applications.

In the present study, an attempt is made to solve these problems simultaneously by using a method similar to that proposed by Stoynov (4-dimensional analysis). The most important assumption is that although the consecutive impedance measurements were performed at different system states, each of the measured impedance values can be accepted as "valid" in the classical sense.

\section{Experimental}

\subsection{Electrodeposition of PEDOT}


Poly(3,4-ethylenedioxytiophene) films were prepared by galvanostatic deposition on both sides of thin gold plates from $0.01 \mathrm{~mol} \cdot \mathrm{dm}^{-3}$ ethylenedioxytiophene (EDOT) solution containing $0.1 \mathrm{~mol} \cdot \mathrm{dm}^{-3} \mathrm{Na}_{2} \mathrm{SO}_{4}$ supporting electrolyte (all the plates were practically identical and were cut from the same gold sheet). Analytical grade 3,4-ethylenedioxythiophene (Aldrich), p.a. $\mathrm{Na}_{2} \mathrm{SO}_{4}$ (Fluka), and ultra-pure water (specific resistance $18.3 \mathrm{M} \Omega \mathrm{cm}$ ) were used for solution preparation. All solutions were purged with oxygen-free argon (Linde 5.0) before use and an inert gas blanket was maintained throughout the experiments.

The deposition was performed in a standard three electrode cell in which the gold plate in contact with the solution served as the working electrode (WE). A spiral shaped gold wire immersed in the same solution served as the counter electrode (CE), and a $\mathrm{KCl}$-saturated calomel electrode (SCE) as the reference electrode (RE). A constant current density of $j=0.2 \mathrm{~mA} \cdot \mathrm{cm}^{-2}(I=0.2 \mathrm{~mA})$ was applied for $1000 \mathrm{~s}$ (the geometric surface area of the working electrode was $A=1.0 \mathrm{~cm}^{2}$ ). The film thickness was estimated from the polymerization charge by using the charge/film volume ratio determined earlier by direct thickness measurements [2224]. The average thickness of the PEDOT film was about $0.8 \mu \mathrm{m}$, the structure of the PEDOT film was globular, cauliflower-like $[9,12,14]$, i.e. the thickness of the film was non-uniform.

\subsection{Cyclic voltammetry and impedance measurements}

Solutions used for cyclic voltammetric and impedance measurements were prepared with ultra-pure water and p.a. $\mathrm{H}_{2} \mathrm{SO}_{4}$ (Merck). The solutions were purged with oxygen-free argon (Linde 5.0) before use and an inert gas blanket was maintained throughout the experiments. In the conventional three-electrode cell configuration the PEDOT-modified gold substrate in contact with the solution was used as the working electrode (WE), a spiral shaped gold wire immersed in the same solution as the counter electrode (CE), and a NaCl-saturated calomel electrode (SSCE) as the reference electrode (RE). The counter electrode was arranged cylindrically around the working electrode to maintain a uniform electric field. After electrodeposition of the films, the PEDOT coated gold plates were rinsed with deionized water to remove monomer molecules. Before the electrochemical experiments (i.e. recording of cyclic voltammograms, overoxidation of the PEDOT film, impedance measurements) the samples were kept in $0.1 \mathrm{~mol} \cdot \mathrm{dm}^{-3} \mathrm{H}_{2} \mathrm{SO}_{4}$ solutions for at least one day in order to remove any residues of the deposition solution. Cyclic voltammetric curves were recorded before and after overoxidation of the Au|PEDOT films in $0.1 \mathrm{~mol} \cdot \mathrm{dm}^{-3}$ sulfuric acid (aq.) in the potential range of $-0.1 \mathrm{~V}$ to $0.6 \mathrm{~V}$ vs. SSCE (sweep rate of $v=50 \mathrm{mV} / \mathrm{s}$ ). The PEDOT films were overoxidized 
by potential cycling in a $0.1 \mathrm{~mol} \cdot \mathrm{dm}^{-3}$ sulfuric acid solution, performing 3 potential cycles between $0.4 \mathrm{~V}$ and $1.5 \mathrm{~V}$ vs. SSCE at a sweep rate of $v=50 \mathrm{mV} / \mathrm{s}$.

After the overoxidation cycles, subsequent impedance measurements were performed at $0.4 \mathrm{~V}$ vs. SSCE. The impedance measurements were performed at 60 discrete frequencies in the frequency range of $0.1 \mathrm{~Hz}-100 \mathrm{kHz}$ at an amplitude of $5 \mathrm{mV}$ rms. 63 successive impedance spectra were recorded. The recording time of a "single" spectrum was about $346 \mathrm{~s}$.

A Zahner IM6 electrochemical workstation (controlled by the Thales software package) was used in all electrochemical experiments.

\subsection{Spline interpolation}

A 3D complex interpolation method using a three-dimensional bicubic spline interpolation algorithm [25] (see also Appendix I.) implemented in LabVIEW was used for the reconstruction of "instantaneous impedances". Mathematically, this corresponds to the 4-dimensional analysis proposed by Stoynov [1-3,6]. As a result a set of instantaneous impedances related to a selected instant of time has been obtained. (The (arbitrarily) selected time instants correspond to the starting times of the frequency scans.)

\section{Results and discussion}

\subsection{Cyclic voltammetry and overoxidation of the PEDOT-film}

Cyclic voltammetric curves recorded before and after overoxidation of a gold $\mid$ PEDOT $\mid 0.1 \mathrm{~mol} \cdot \mathrm{dm}^{-3}$ sulfuric acid (aq.) electrode (practically identical to that used in the impedance measurements) are presented in Fig. 1.

Fig. 1.

The hysteresis-free quasi-rectangular shape of the CV curve recorded before overoxidation indicates an almost purely capacitive behavior of the electrode. The voltammograms recorded before and after overoxidation (curves 1 and 2-5 in Fig. 1) are similar in shape and show typical (pseudo-)capacitive behavior at the narrow voltage window ( $-0.1 \mathrm{~V}$ to $0.6 \mathrm{~V}$ vs. SSCE), but the redox capacity of the (over)oxidized polymer film is considerably smaller than that of the freshly prepared film. The small but distinct differences in the shapes of the curves immediately after the reversal of the direction of the potential scan imply that the resistance of the system 
changes with time. In addition, we can infer from the decay rates of the current ("time constants") that the resistance is decreasing.

The potential program applied to the PEDOT-modified electrode used in the impedance measurements (geometric surface area: $1.0 \mathrm{~cm}^{2}$ ) is given in Fig. 2a, and the corresponding cyclic voltammograms in Fig. 2 b.

Fig. 2.

In the 3 overoxidation cycles, the positive potential limit has been set to $1.5 \mathrm{~V}$ vs. SSCE (Fig. 2a). It is known [9-14,21] that between -0.3 and $0.8 \mathrm{~V}$ vs. SSCE the oxidation-reduction process of the PEDOT films is reversible. However, at more positive potentials irreversible degradation of the polymer layer occurs. As it can be seen in Fig. 1 and in Fig. 2b (curve O), the cyclic voltammograms of PEDOT-modified electrodes show almost pure capacitive behavior in the potential range between $-0.1 \mathrm{~V}$ and $+0.6 \mathrm{~V}$, i.e. if the positive potential limit is kept below $0.8 \mathrm{~V}$ vs. SSCE. If the polarization potential exceeds this critical value an oxidation peak without corresponding reduction peak appears (see Fig. 2b) [9-14]. It is (somewhat arbitrarily) assumed that the overoxidaton process stopped completely at $\tau_{1}$ (i.e. at $t=-53.0 \mathrm{~s}$ ), consequently, $\Delta \tau=53.0 \mathrm{~s}$.

\subsection{Impedance measurements}

In accordance with Fig.2a the impedance measurements started at $\tau_{2}$. This moment was taken as the starting time of the impedance measurements ("timestamp": $t=0 \mathrm{~s}$ ).

Fig. 3.

In Fig. 3 the successive impedance spectra recorded at $0.4 \mathrm{~V}$ vs. SSCE after the overoxidation cycles over a frequency range from $0.1 \mathrm{~Hz}$ to $100 \mathrm{kHz}$ are presented in the complex plane, as Bode diagrams (i.e. as $\log |Z|$ vs. $\log f$ and $-\phi$ vs. $\log f$ plots, where $|Z|$ is the magnitude of the impedance, $f$ is the frequency and $\phi$ is the phase angle, Fig. 3c-d) and as $\log$ ("wparallel capacitance") vs. $\log f$ plots (Fig. 3b). The latter are in fact $\log (\operatorname{Im}(Y) / \omega)$ vs. $\log f$ plots, where the admittance $Y$ is the reciprocal value of the impedance corrected for the uncompensated ohmic resistance $\left(Z_{\text {corr }}\right)$. The most interesting feature in Fig. $3 a$ is the continuous decrease of the diameter of the high-frequency capacitive arc with time. On the other hand, it can be seen in 
Fig. $3 \mathrm{~b}$ that while the low frequency capacitance is practically independent of time, the high frequency capacitance slowly decreases during the experiment. Apparently, the high and medium frequency regions of the impedance spectra are stronger affected by the time evolution than the values measured at low frequencies (see. Figs 3b-c).

\subsection{The 3-dimensional representation of the measured data the 4-dimensional analysis}

The 3D representation of the measured data is shown in Fig. 4. The complex plane diagrams in Figs. 3 a and 4 are similar to those reported for other polymer modified electrodes [26,27]. The impedance data show a capacitive arc ("depressed semicircle") in the highfrequency region and a (CPE-like) capacitive domain at low frequencies [28]. On the other hand, no significant Warburg region can be seen in the spectra [29]. This can be explained by the facts that the films are relatively thin, the conductivities (electronic and ionic) are high, and the film thickness is highly non-uniform, especially after overoxidation. The effect of film thickness and thickness distribution on the impedance of polymer-modified electrodes was discussed by Inzelt et al. and by Gabrielli et al. as early as 1991 [30,31]. On the basis of computer simulations it was predicted, that if the film/electrolyte solution surface is very rough, i.e., the film consists of very thin and thick regions, no Warburg section will appear in the impedance spectra, the low frequency capacitance $\left(C_{\mathrm{L}}\right)$ will be frequency dependent, and the broader the thickness distribution the higher the degree of the frequency dispersion of $C_{\mathrm{L}}$. (The results are well illustrated in the relevant figures in refs. [9,27,30-31].)

Fig. 4.

Since the successive impedance diagrams are continuously changing with time (see Figs. 3 and 4), it is evident that the system is still in a transient state rather than in a stationary (or equilibrium) state. As already discussed in the introduction, in such cases a postexperimental analytical procedure is necessary for the reconstruction of "instantaneous impedances" [2,3,6,8,32-34]. In this study a 3D interpolation method using a three-dimensional bicubic spline interpolation algorithm has been adapted ([25], Appendix I.). Two different approaches were applied for the calculation of the corrected (synthetic) impedance diagrams. In the first, the measured magnitudes and phase angles were used in the interpolation/extrapolation process (Fig. 5), and the interpolated/extrapolated magnitude and phase angle values corresponding to the same time moments were converted into real and 
imaginary parts of the complex impedance. In the second, the interpolation/extrapolation was carried out using real and imaginary parts of impedances measured at identical frequencies (,isofrequential components”) [1] (see Fig. 6). This means that for every measured frequency two one-dimensional functions of iso-frequency dependencies (for the real and for the imaginary components) have been constructed, and on the basis of the continuity of the evolution, interpolation (and extrapolation) has been performed. As a result a set of "instantaneous" impedances related to a selected instant of time (i.e. the beginning of each frequency can) has been obtained.) As it can be seen by comparing Figs 5 and 6, the corrected impedances obtained by the two methods practically coincide.

Fig.5.

Fig.6.

According to the above considerations, interpolation and/or extrapolation allows the reconstruction of "instantaneous" spectra from a series of time-dependent spectra. The "instantaneous" (calculated) spectra can be regarded as undistorted. By extrapolation to $t=0$ ( $\tau_{2}$ in Fig. 2 ) the impedance diagram corresponding to the starting time of the measurement series could be constructed (plots designated by S1 in Figs. 7a and 7b). Diagrams 1 in Figs. 7a and $7 \mathrm{~b}$ show the experimental ("original") data points measured in the "frequency by frequency" mode of operation, i.e. during the frequency sweep which started at $\tau_{2}(t=0)$ in Fig. 2. The next two measured spectra (in case of plots 2 and 3 the measurements started at $t=346.6 \mathrm{~s}$ and $t=692.5 \mathrm{~s}$ ) and the calculated "instantaneous" spectra (S2 and S3, calculated values for $t=346.6 \mathrm{~s}$ and $t=692.5 \mathrm{~s}$ ) are also shown in Figs. 7a and 7b. (Diagrams 1-3 are the first three sets of data measured in the series and contains inherent time dependence.) Bode plots (in the relevant frequency range) corresponding to diagrams 1,2,S1 and S2 in Fig. 7a-7b are given in Figs. $7 \mathrm{c}$ and $7 \mathrm{~d}$.

Fig.7.

All the above results imply that the 4-dimensional analysis method can not only be used for the correction of experimental data (i.e. the measured impedances), but it opens up the possibility of the estimation of the impedance spectra outside the time interval of the impedance measurements. Complex plane and Bode plots of the calculated impedance corresponding to 
the presumed end of the overoxidation process (at $t=-53.0 \mathrm{~s}$, see Fig. 2) are shown in Fig. 7ad (plots marked by E). All these data points have been obtained by spline extrapolation outside the range of the measured data.

\subsection{CNLS estimation of the parameters}

One of the most general theoretical models for polymer modified electrodes involving coupled diffusion-migration charge transport mechanism with three charge carriers has been derived in [35]. The essential feature of this model is the assumption of a coupling of the oscillation amplitude of the concentration for the charge carriers. The polymer film is supported on a metal or other electronic conductor and dipped in an electrolyte solution containing only electrochemically "inert" species, i.e., the solution only contains ions which do not possess a redox activity ("background electrolyte"). In this electrode arrangement two different interfaces exist: a metal/film interface where only electrons may be exchanged and a film/electrolyte solution interface which is permeable only for counterions, which are able to cross the film/solution boundary to retain the bulk film electroneutrality, and for neutral (e.g. solvent) molecules ("blocking" interface). The general expression of the impedance taking into account the uncompensated ohmic resistance $\left(R_{\mathrm{u}}\right)$ and two interfacial capacitances at the two interfaces $\left(C_{1}\right.$ and $\left.C_{3}\right)$ is given by

$$
Z(\omega)=R_{\mathrm{u}}+Z_{2}(\omega)+\frac{1}{1 / Z_{1}(\omega)+\mathrm{i} \omega C_{1}}+\frac{1}{1 / Z_{3}(\omega)+\mathrm{i} \omega C_{3}}
$$

where

$$
\begin{aligned}
& Z_{1}(\omega)=R_{11}+R_{12} \frac{\operatorname{coth} \frac{s d}{2}}{s}+R_{13} \frac{\tanh \frac{s d}{2}}{s}, Z_{2}(\omega)=R_{21}+R_{23} \frac{\tanh \frac{s d}{2}}{s}, \\
& Z_{3}(\omega)=R_{31}+R_{32} \frac{\operatorname{coth} \frac{s d}{2}}{s}+R_{33} \frac{\tanh \frac{s d}{2}}{s}, \text { and } s^{2}=\sqrt{i \omega D^{*}}
\end{aligned}
$$

In the above equations $\mathrm{i}$ is the imaginary unit, $d$ is the film thickness, $\omega$ is the angular frequency, $D^{*}$ is an "effective" diffusion coefficient, and the $R_{\mathrm{jk}}-\mathrm{s}(\mathrm{j}, \mathrm{k}=1, \ldots, 3)$ are frequency independent quantities, respectively. It follows from the model that $R_{11}$ and $R_{31}$ can be identified as the charge transfer resistances at the two phase boundaries $\left(R_{11}\right.$ is the charge transfer resistance at the metal|polymer interface, while $R_{31}$ is the charge transfer resistance at the polymer film|solution interface), and $R_{21}$ is a pure ohmic resistance depending on the film thickness. The simplification of the general formula results in similar impedance expressions that have been 
derived in several papers for two mobile charge carriers and diffusion or diffusion-migration transport. Nevertheless, it is well known that the 'ideal' impedance response, i.e., a separate Randles circuit behavior at high frequencies, a Warburg section at intermediate frequencies, and a purely capacitive behavior due to the redox capacitance at low frequencies seldom appears in real systems [27,30,31]. This is also true for PEDOT-modified electrodes. The deviations of the impedance responses of polymer modified electrodes from those predicted by the theories have been explained by taking into account different effects such as interactions between redox sites, ionic relaxation processes, distribution of diffusion coefficients, inhomogeneous oxidation/reduction processes, uneven film thickness, etc. (see e.g. [26]). Apparently in case of PEDOT modified electrodes the most important cause of the deviations is the uneven thickness distribution of the polymer layer. Because of the cauliflower-like structure [9,12-14] the film thickness is highly nonuniform. In addition, in case of overoxidized PEDOT films incomplete coverage of the film on the substrate can be observed in some areas [9]. Thus, it is unlikely to get ideal capacitive response at (not extremely) low frequencies and the Warburg section becomes distorted. The effect of the film thickness and the thickness distribution on the impedance response of poly(tetracyanoquinodimethane) (PTCNQ) modified electrodes was discussed e.g. in [29]. It was shown that the high frequency part the measured impedance spectra is in good agreement with the theoretical model, but for the proper description of the "whole" impedance plot the thickness distribution of the polymer film should be taken into account. However, the thickness distribution function is usually unknown. The finding that the high frequency part of the impedance spectra is independent of the thickness distribution is in complete accordance with the more general results reported in [30]. However, this is not too surprising since at sufficiently high frequencies the impedances $Z_{1}(\omega), Z_{2}(\omega)$ and $Z_{3}(\omega)$ can be well approximated by $R_{11}, R_{21}$ and $R_{31}$, respectively. Since $R_{21}$ and $R_{31}$ are usually negligibly small compared to $R_{11}$ (and $C_{3}$ is very small) the overall impedance can be represented fairly accurately by the approximate expression

$$
Z(\omega) \approx R_{\mathrm{u}}+\frac{1}{1 / R_{11}+\mathrm{i} \omega C_{1}} .
$$

However, unfortunately, the situation is more complicated than just described. One thing to consider is the frequency dependence of the high frequency capacitance that is usually observed for polymer modified electrodes (and was also observed in the present case). In some relevant papers the dispersion of the high-frequency capacitance has been attributed to the microscopic roughness of the electrode surface [36-39] and an adsorption pseudocapacitance connected with 
the charging / discharging process within the first layer of the film at the metal interface [40]. Although in the case of overoxidized PEDOT film electrodes the physicochemical origin of the frequency dispersion is rather uncertain, it seems to be a plausible assumption, that the distributed polymer/solution interface does not respond as an ideal capacitor, and it may be represented by a constant-phase element (CPE) with an exponent $0<\alpha \leq 1$, ( $\alpha$ is usually close to 1$)$. The impedance of this element is $Z_{\mathrm{CPE}}(\omega)=\frac{1}{B}(\mathrm{i} \omega)^{-\alpha}$, where $\mathrm{i}$ is the imaginary unit, $B$ and $\alpha$ are the CPE parameters, and $\omega$ is the angular frequency, respectively.

In accordance with the above considerations, the values of the charge transfer resistances at $t=0 \mathrm{~s}$ and at some other time instants were estimated by fitting the highfrequency part of the "instantaneous" (calculated) spectra with the impedance function (Eq. 3) corresponding to the equivalent-circuit analog shown in the inset of Fig. 8 a.

$$
Z(\omega)=R_{\mathrm{u}}+\frac{1}{R_{\mathrm{ct}}^{-1}+B(\mathrm{i} \omega)^{\alpha}}
$$

In Eq. $3 R_{\mathrm{ct}}$ is the charge transfer resistance (corresponding to $R_{11}$ in Eqs. 1 and 2) and $R_{\mathrm{u}}$ is the uncompensated ohmic resistance (which is mainly the resistance of the electrolyte solution between the working and the reference electrode).

The estimated values of the parameters (obtained by complex non-linear least squares (CNLS) fitting program based on the Gauss-Newton-Levenberg-Marquardt method [41-43] with Poisson-weighting) are shown in Table. 1 (the statistics of the parameter estimation is given in Table 2). Though the variances are unknown, considering the small residuals the fit can be intuitively judged acceptable [27,36]. Indeed visual inspection also supports this conclusion (the diagrams shown in Fig. 8 can be used for visual inspection of the fits). E and S1-S5 in Fig. 8a are the calculated "instantaneous impedances", corresponding to $t=-53.0 \mathrm{~s}$, $t=0 \mathrm{~s}, t=346.6 \mathrm{~s}, t=692.5 \mathrm{~s}, t=1039.4 \mathrm{~s}$, and $t=1386.7 \mathrm{~s}$, respectively. The solid curves were calculated (simulated) by using the "best-fit" parameters given in Table 1. The corresponding Bode plots in the relevant frequency ranges are given in Figs. 8b and 8c.

Fig.8.

Fig.9.

Some of the charge transfer resistance values obtained by CNLS-fitting of the “instantaneous" impedances are shown in Fig. 9. It can be seen from Table 1 (and from Fig. 9), 
that the $R_{\mathrm{ct}}$ value corresponding to the time instant just after overoxidation of the film is about $38.5 \Omega$. Starting from this value, $R_{\mathrm{ct}}$ decreases continuously with experiment time to a value somewhat higher than the charge transfer resistance of the pristine electrode.

(Note: It was assumed in this study that each of the impedance values measured during consecutive measurements can be accepted as "valid" in the classical sense. However, even in this case the measured series of impedances do not belong to a "true" impedance spectrum and the measured set of data should not be used for traditional impedance analysis in the frequency domain (e.g. by relating the experimental "impedance spectra" to the underlying physics of the system by fitting an equivalent circuit analog). Disregarding the fact that such a procedure is physically unjustified in the present case (the system is nonstationary, and it is known that in case of nonstationarity a CNLS analysis may result in spurious, distorted or even unphysical parameter estimates), for the sake of comparison we applied the CNLS fitting method to the high and medium frequency part of the data set obtained directly from the measurement that started at time $t=0$. The resulting estimated parameters are $R_{\mathrm{u}} / \Omega=7.471, R_{\mathrm{ct}} / \Omega=3.602$, $\alpha=0.930$, and $B /\left(\Omega^{-1} \mathrm{~S}^{\alpha}\right)=1.295 \cdot 10^{-5}$, respectively. These results are suggesting that the charge transfer resistance is about $3.5 \%$ smaller than its true value and the frequency dispersion of the capacitance is considerably lower than it is in reality. Although the absolute differences may appear relatively small the systemic deviations of the parameter estimates from the "true" values may result in serious misinterpretations and acceptance of essentially unreliable data, especially if the complete impedance function is fitted to the experimental values in the whole range of frequencies.)

A possible explanation for the decrease of the charge transfer resistance with time is that after overoxidation the number of the polymer chains directly contacted with (adsorbed on) the substrate surface is increasing with the passing time. During overoxidation at sufficiently positive potentials a significant fraction of the polymer chains become detached from the substrate surface (i.e. "desorption", "deactivation" or "delamination" occurs). On the other hand, at a less positive potential the readsorption of the polymer chains (polymer chain ends) becomes possible. This means that during overoxidation the "effective" coverage of the substrate by the polymer decreases, and at less positive potentials the coverage may start to increase again. The direction of the change in $R_{\mathrm{ct}}$ is in agreement with this hypothesis. The above considerations are supported by recent results reported in ref. [44] according to which the decrease of the charge transfer resistance was observed with an increase in amount of deposited Prussian Blue and nickel hexacyanoferrate films. The authors of [44] explained the 
observed trend in the charge transfer resistance during the deposition of the electroactive films as follows: At zero coverage the charge transfer resistance at the substrate | solution interface is very high (in principle, for an ideally polarizable electrode where faradaic reactions cannot occur, $\left.R_{\mathrm{ct}}=\infty[45,46]\right)$. At low amount of the film deposited, the electrode is only partially covered with the film. When the electrode is only partially covered, the area of the electrode | film interface increases as the amount of the deposited inorganic film increase causing the decrease of the charge transfer resistance. In addition, it was shown in [44] by scanning electron microscopy (SEM) that the charge transfer resistance evaluated from impedance spectra represents the degree of surface coverage.

\section{Conclusions}

The impedance spectra of overoxidized PEDOT films differ from those measured for freshly prepared films. The decreasing capacitance and the increasing resistance suggest that during overoxidation the electrochemical activity of the film decreases and the charge transfer process at the metal/film interface becomes more hindered than in the case of pristine films. The time evolution of the impedance spectra is a remarkable feature of the "overoxidized" PEDOT modified electrodes. If the electrode potential is held in the "stability region" of the film after overoxidation, the impedance spectra change continuously over several hours, i.e. the system is intrinsically nonstationary and is affected by time-dependent phenomena.

The 4-dimensional analysis method, originally proposed by Stoynov, can serve as an efficient tool for the study of non-stationary systems. The method was successfully applied for the determination of the charge transfer resistance and some other characteristic impedance parameters of the gold $|\mathrm{PEDOT}| 0.1 \mathrm{~mol} \cdot \mathrm{dm}^{-3}$ sulfuric acid (aq) electrode corresponding to different time instants, including the time instant just after the overoxidation of the polymer film. This means that the 4-dimensional analysis method can not only be used for the correction of the existing (experimentally measured) impedance data, but it opens up the possibility of the estimation of the impedance spectra outside the time interval of the impedance measurements.

According to the results the high and medium frequency regions of the impedance spectra are apparently stronger affected by the time evolution than the values measured at low frequencies. Since the low frequency capacitance $\left(C_{\mathrm{L}}\right)$ is almost time independent, the changes of the impedance spectra with time can be assumed to be entirely due to time evolution of the charge transfer resistance $\left(R_{\mathrm{ct}}\right)$ and the double layer capacitance $\left(C_{\mathrm{dl}}\right)$ at the gold substrate/polymer interface. 
In the present experiments, starting from a maximum value of about $38.5 \Omega \cdot \mathrm{cm}^{2}$, the charge transfer resistance decreased continuously with experiment time to a value somewhat higher than the $R_{\mathrm{ct}}$ of the pristine electrode, implying that some "self-healing process" occurred at the film/substrate interface. Unfortunately, the exact mechanism of this phenomenon is still not known, but it may be related to the slow readsorption of the polymer chains after overoxidation of the film.

\section{Acknowledgements}

Support from the Hungarian Scientific Research Fund, the National Research, Development and Innovation Office - NKFI (grants No. K 109036, VEKOP-2.3.2-16), and from the Russian Foundation for Basic Research (grant N-16-03-00457) is gratefully acknowledged.

\section{Appendix I.}

Experiments often result in a sequence $\left\{\left(x_{i}, y_{i}\right), i=1,2, \ldots, m\right\}$ of data pairs and it is assumed that there exists a functional relationship $y=f(x)$ between the two variables. The discrete points $\left(x_{1}, \ldots, x_{m}\right)$ and $\left(y_{1}, \ldots, y_{m}\right)$ are referred to as grid points ("grid nodes") and function values, respectively. However, in practice the form of this function is often unknown. In some other cases the functional form can be deduced from physical principles, but it is too complex for meaningful parameter estimation. In both situations one can try to predict (estimate) some properties of $f(x)$ directly from the measured values $\left(x_{i}, y_{i}\right)$, e.g.: a) the function value $f(x)$ between two grid points (interpolation); b) the derivative $f^{\prime}(x)$ (numerical differentiation); and c) the integral $\int_{x_{a}}^{x_{b}} f(x) \mathrm{d} x$, where $x_{1} \leq x_{\mathrm{a}}<x_{\mathrm{b}} \leq x_{m}$ (numerical integration).

Since the function values are known only at the grid points, to solve these problems we need to connect the data points by some plausible interpolating function. Its form should be sufficiently general to approximate a wide range of nonlinear relationships (large classes of functions), but simple enough to deal with. The most common among such functions are polynomials. The interpolation is called global if all data pairs are used simultaneously. The interpolation is local if only $n<m$ grid points around the point $x_{j}$ of interest. The most frequently used interpolation methods are local linear, quadratic and cubic interpolation (i.e., $n=2, n=3$ and $n=4$, respectively). Nevertheless, it is known that local cubic interpolation results in a function whose 
derivative is not necessarily continuous at the grid points. With a non-1ocal adjustment of the coefficients we can, however, achieve global differentiability up to the second derivatives. Such functions, still being cubic polynomials between each pair of grid points, are called cubic splines.

In case of spatial interpolation, the point-value pairs are located in a three dimensional space. The data are structured, if the known sampling points are located in a two dimensional grid. Given the function values at grid points $f\left(x_{i}, y_{i}\right)=u_{i j}$ for $i=0, \ldots, \mathrm{m} ; j=0, \ldots, n$, with interpolation it is possible to estimate the function value at an arbitrary point $(x, y)$ inside the grid. Therefore a function that passes through the given function values is defined.

Bicubic spline interpolation extends cubic spline interpolation to two dimensional data: With cubic splines, which are calculated for all rows and columns, the values of the partial derivatives may be approximated at the sampling points. In other words, the bicubic spline interpolation method divides the testing area into intervals between the sampling points, and assumes a third degree polynomial on each interval. The separate functions have to blend smoothly into each other at the interval boundaries, hence the first and second derivatives of the neighboring functions have to coincide. With these approximated values and the known values at the sampling points the coefficients $a_{i j}$ of the generally bicubic function $f(x, y)=\sum_{i, j=0, \ldots, 3} a_{i j} x^{i} y^{j}$ may be calculated for every grid cell.

In practice the bicubic spline interpolation is carried out by two successive interpolations according to direction $X$ then $Y$. The function values are defined in nodes of a Cartesian grid $u_{i}=u\left(x_{i}\right)$, for $i: 1, \ldots, m$ and each value $u$ in $X$ location is computed by the following formulation according to a direction $X$ then $Y$ :

$$
u=A u_{j}+B u_{j+1}+C u_{j}^{\prime \prime}+D u_{j+1}^{\prime \prime}
$$

where

$$
A=\frac{x_{j+1}-x}{x_{j+1}-x_{j}}, B=\frac{x-x_{j}}{x_{j+1}-x_{j}}, C=\frac{1}{6}\left(A^{3}-A\right)\left(x_{j+1}-x_{j}\right)^{2} \text {, and } D=\frac{1}{6}\left(B^{3}-B\right)\left(x_{j+1}-x_{j}\right)^{2} \text {. }
$$

We take derivatives of equation (A1) with respect to $x$, using the definitions of $A, B, C, D$ to compute $d A / d x, d B / d x, d C / d x$ and $d D / d x$. The result is

$$
u^{\prime}=\frac{u_{j+1}-u_{j}}{x_{j+1}-x_{j}}-\frac{3 A^{2}-1}{6}\left(x_{j+1}-x_{j}\right) u_{j}^{\prime \prime}+\frac{3 B^{2}-1}{6}\left(x_{j+1}-x_{j}\right) u_{j+1}^{\prime \prime}
$$

for the first derivative, and 


$$
u^{\prime \prime}=A u_{j}^{\prime \prime}+B u_{j+1}^{\prime \prime}
$$

for the second derivative.

Since $A=1$ at $x_{j}, A=0$ at $x_{j+1}$, while $B=1$ at $x_{j+1}$, and $B=0$ at $x_{j}$, equation (A3) shows that $u^{\prime \prime}$ is just the tabulated second derivative, and also that the second derivative will be continuous across (e.g.) the boundary between the two intervals $\left(x_{\mathrm{j}-1}, x_{\mathrm{j}}\right)$ and $\left(x_{\mathrm{j}}, x_{\mathrm{j}+1}\right)$. The fundamental idea of a cubic spline is to require that the first derivative, computed from equation (A2), be continuous across the boundary between two intervals.

The required equations are obtained by setting equation (A2) evaluated for $x=x_{j}$ in the interval $\left(x_{j}-1, x_{j}\right)$ equal to the same equation evaluated for $x=x_{j}$ but in the interval $\left(x_{j}, x_{j+1}\right)$. This gives after some rearrangement (for $j=2, \ldots, m-1$ ):

$$
\frac{x_{j}-x_{j-1}}{6} u_{j-1}^{\prime \prime}+\frac{x_{j+1}-x_{j-1}}{3} u_{j}^{\prime \prime}+\frac{\left(x_{j+1}-x_{j}\right)}{6} u_{j+1}^{\prime \prime}=\frac{u_{j+1}-u_{j}}{x_{j+1}-x_{j}}-\frac{u_{j}-u_{j-1}}{x_{j}-x_{j-1}}
$$

Thus we have $m-2$ equations in the $m$ unknowns $u_{i}^{\prime \prime}$, and need two further constraints for a unique solution. In most cases these are chosen according to one of the following alternatives: i) Assume that one or both second derivatives vanish at the end points $x_{1}$ and $x_{m}$, resulting in a function called "natural cubic spline".

ii) The first derivative has arbitrarily fixed values on either or both boundaries (i.e. at one or both end points).

It can be verified that the set of linear equations given by the constraints has a unique solution both for cases i) and ii), if the grid points $x_{1}, \ldots, x_{m}$ are distinct. 
Table 1.

Results of CNLS fitting of impedance data obtained using the 4-dimensional analysis method ("instantaneous impedances"): the estimated values of the parameters. $R_{\mathrm{u}}$ is the uncompensated ohmic resistance (resistance of the solution), $R_{\mathrm{ct}}$ is the charge transfer resistance, $B$ and $a$ are the parameters of the CPE, $\Delta R_{\mathrm{u}}, \Delta R_{\mathrm{ct}}, \Delta B$ and $\Delta \alpha$ are the corresponding $95 \%$ confidence intervals.

\begin{tabular}{|c|c|c|c|c|c|c|c|c|c|c|}
\hline $\begin{array}{c}\text { data } \\
\text { set }\end{array}$ & $t / \mathrm{s}$ & $R_{\mathrm{u}} / \Omega$ & $\Delta R_{\mathrm{u}} / \Omega$ & & $R_{\mathrm{ct}} / \Omega$ & $\Delta R_{\mathrm{ct}} / \Omega$ & $B /\left(\Omega^{-1} \mathrm{~s}^{\alpha}\right)$ & $\Delta B /\left(\Omega^{-1} \mathrm{~s}^{\alpha}\right)$ & $\alpha$ & $\Delta \alpha$ \\
\hline E & -53.0 & 7.42 & \pm 0.13 & 38.49 & \pm 0.68 & $1.294 \cdot 10^{-5}$ & $\pm 0.056 \cdot 10^{-5}$ & 0.920 & \pm 0.010 \\
\hline S1 & 0 & 7.39 & \pm 0.11 & 37.29 & \pm 0.54 & $1.213 \cdot 10^{-5}$ & $\pm 0.044 \cdot 10^{-5}$ & 0.9126 & \pm 0.0090 \\
\hline S2 & 346.6 & 7.37 & \pm 0.12 & 29.09 & \pm 0.36 & $9.58 \cdot 10^{-6}$ & $\pm 0.37 \cdot 10^{-6}$ & 0.9138 & \pm 0.0093 \\
\hline S3 & 692.5 & 7.50 & \pm 0.14 & 22.96 & \pm 0.35 & $8.93 \cdot 10^{-6}$ & $\pm 0.44 \cdot 10^{-6}$ & 0.908 & \pm 0.012 \\
\hline S4 & 1039.4 & 7.47 & \pm 0.12 & 18.60 & \pm 0.28 & $8.49 \cdot 10^{-6}$ & $\pm 0.42 \cdot 10^{-6}$ & 0.902 & \pm 0.012 \\
\hline S5 & 1386.7 & 7.44 & \pm 0.11 & 15.46 & \pm 0.23 & $8.17 \cdot 10^{-6}$ & $\pm 0.39 \cdot 10^{-6}$ & 0.897 & \pm 0.012 \\
\hline S6 & 1765.0 & 7.436 & \pm 0.098 & 12.98 & \pm 0.21 & $7.91 \cdot 10^{-6}$ & $\pm 0.36 \cdot 10^{-6}$ & 0.894 & \pm 0.012 \\
\hline S7 & 2113.5 & 7.415 & \pm 0.087 & 11.28 & \pm 0.18 & $7.70 \cdot 10^{-6}$ & $\pm 0.34 \cdot 10^{-6}$ & 0.891 & \pm 0.012 \\
\hline S8 & 2464.7 & 7.375 & \pm 0.069 & 9.97 & \pm 0.14 & $7.56 \cdot 10^{-6}$ & $\pm 0.29 \cdot 10^{-6}$ & 0.888 & \pm 0.011 \\
\hline S9 & 2823.9 & 7.361 & \pm 0.061 & 8.91 & \pm 0.13 & $7.40 \cdot 10^{-6}$ & $\pm 0.26 \cdot 10^{-6}$ & 0.886 & \pm 0.011 \\
\hline S10 & 3173.1 & 7.372 & \pm 0.067 & 8.16 & \pm 0.17 & $7.07 \cdot 10^{-6}$ & $\pm 0.31 \cdot 10^{-6}$ & 0.876 & \pm 0.014 \\
\hline & & & & & & & & & & \\
\hline
\end{tabular}

Table 2.

Results of CNLS fitting of impedance data obtained using the 4-dimensional analysis method: statistical parameters of the fitting procedure.

\begin{tabular}{|c|c|c|c|c|}
\hline $\begin{array}{c}\text { data } \\
\text { Set }\end{array}$ & $\begin{array}{c}\text { weighted sum of } \\
\text { squares (objective } \\
\text { function) } / 10^{-4}\end{array}$ & degrees of freedom & standard error / 10 & $\begin{array}{c}\text { critical t-value at 95\% } \\
\text { confidence level }\end{array}$ \\
\hline E & 141.142 & 21 & 6.72103 & 2.08233 \\
\hline S1 & 96.2320 & 21 & 4.58248 & 2.08233 \\
\hline S2 & 95.5885 & 21 & 4.55183 & 2.08233 \\
\hline S3 & 113.908 & 20 & 5.69538 & 2.08723 \\
\hline S4 & 82.0470 & 19 & 4.31826 & 2.09270 \\
\hline S5 & 57.1061 & 18 & 3.17256 & 2.09916 \\
\hline S6 & 35.2277 & 16 & 2.20173 & 2.11665 \\
\hline S7 & 24.2635 & 15 & 1.61756 & 2.12844 \\
\hline S8 & 13.5335 & 14 & 0.966679 & 2.14273 \\
\hline S9 & 8.93979 & 13 & 0.687676 & 2.15989 \\
\hline S10 & 7.63755 & 11 & 0.694322 & 2.20488 \\
\hline
\end{tabular}




\section{Figures}

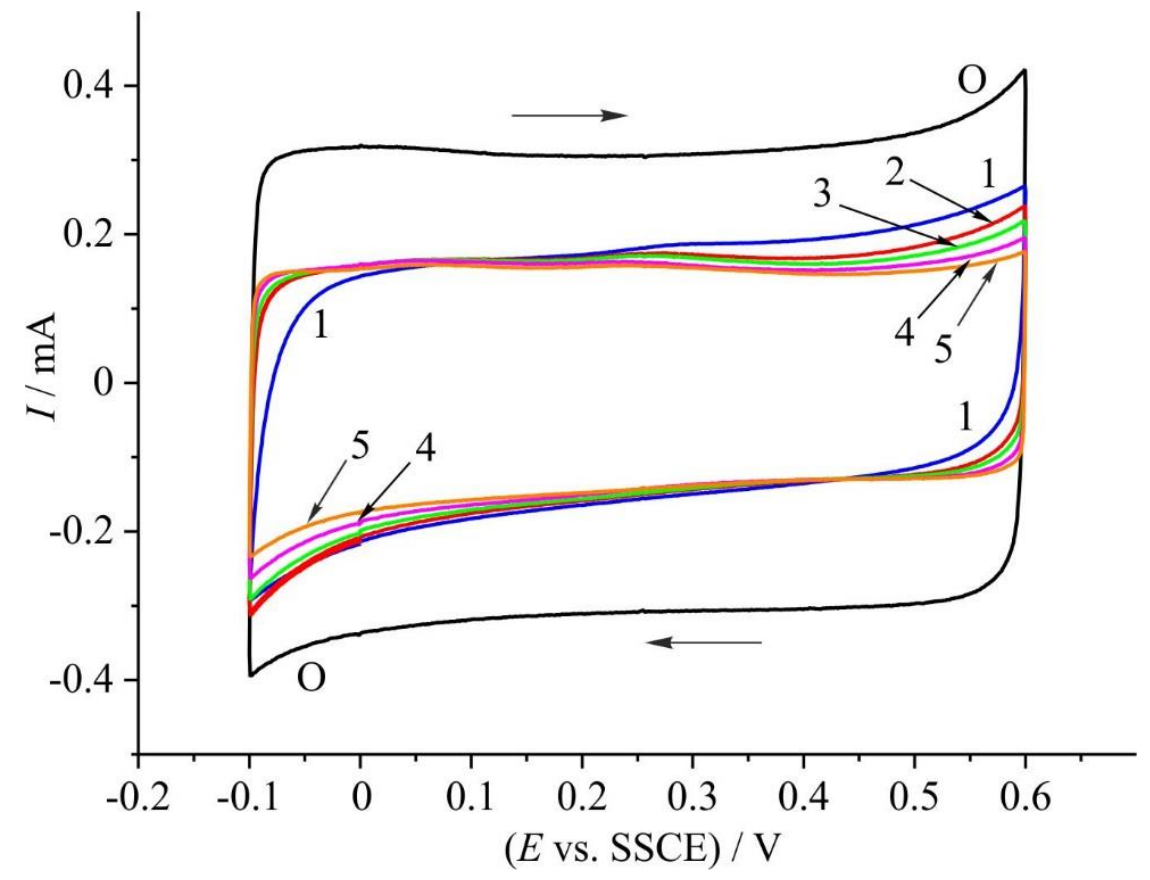

Fig. 1. Cyclic voltammograms of a PEDOT film electrodeposited on gold recorded in $c=0.1 \mathrm{~mol} \cdot \mathrm{dm}^{-3} \mathrm{sulfuric}$ acid solution at a sweep rate of $v=50 \mathrm{mV} \cdot \mathrm{s}^{-1}$. O: typical $\mathrm{CV}$ recorded before overoxidation ("pristine" film). 15: CV curves taken after 3 "overoxidation cycles" applied between $-0.1 \mathrm{~V}$ and $1.5 \mathrm{~V}$ vs. SSCE (see Fig. 2): 1 : after $1 \mathrm{~min}, 2$ : after $10 \mathrm{~min}, 3$ : after $23 \mathrm{~min}$, 4: after $124 \mathrm{~min}$, 5: after $308 \mathrm{~min}$, respectively. Geometric electrode area: $1.0 \mathrm{~cm}^{2}, E$ : electrode potential, $I$ : current.

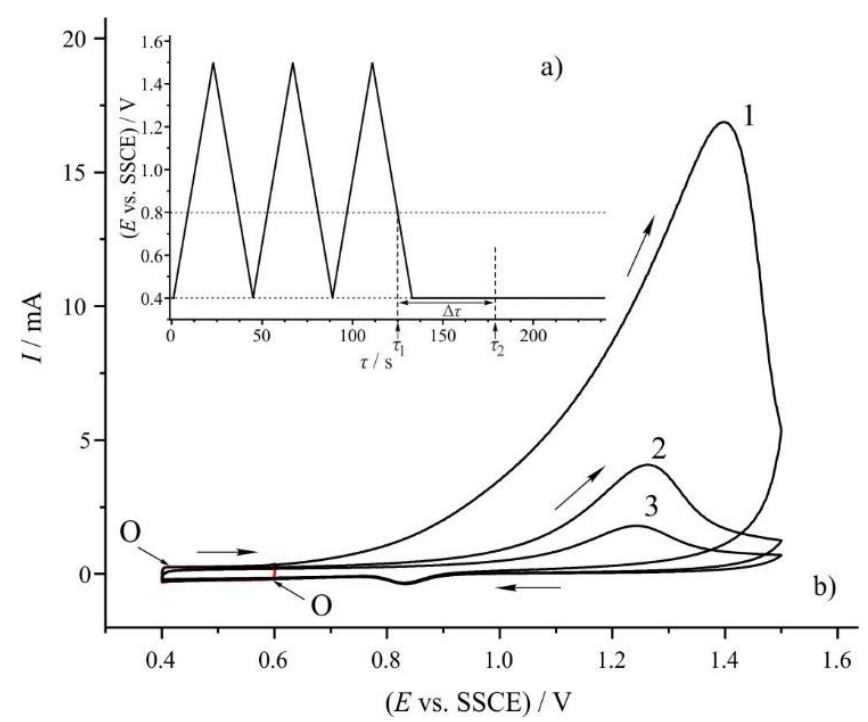

Fig. 2. a) The potential program applied to the PEDOT-modified electrode. $\tau_{1}$ is the estimated end of the overoxidaton process. The impedance measurements started at $\tau_{2} . \Delta \tau=53.0 \mathrm{~s}$.

b) O: Cyclic voltammogram recorded before overoxidation in the potential range between $0.4 \mathrm{~V}$ and $0.6 \mathrm{~V}$ vs. SSCE, sweep rate: $v=50 \mathrm{mV} / \mathrm{s} .1-3$ : Successive cyclic voltammograms (,overoxidation cycles”) recorded in the potential range from $0.4 \mathrm{~V}$ to $1.50 \mathrm{~V}$ vs. SSCE, sweep rate: $v=50 \mathrm{mV} / \mathrm{s}$. 

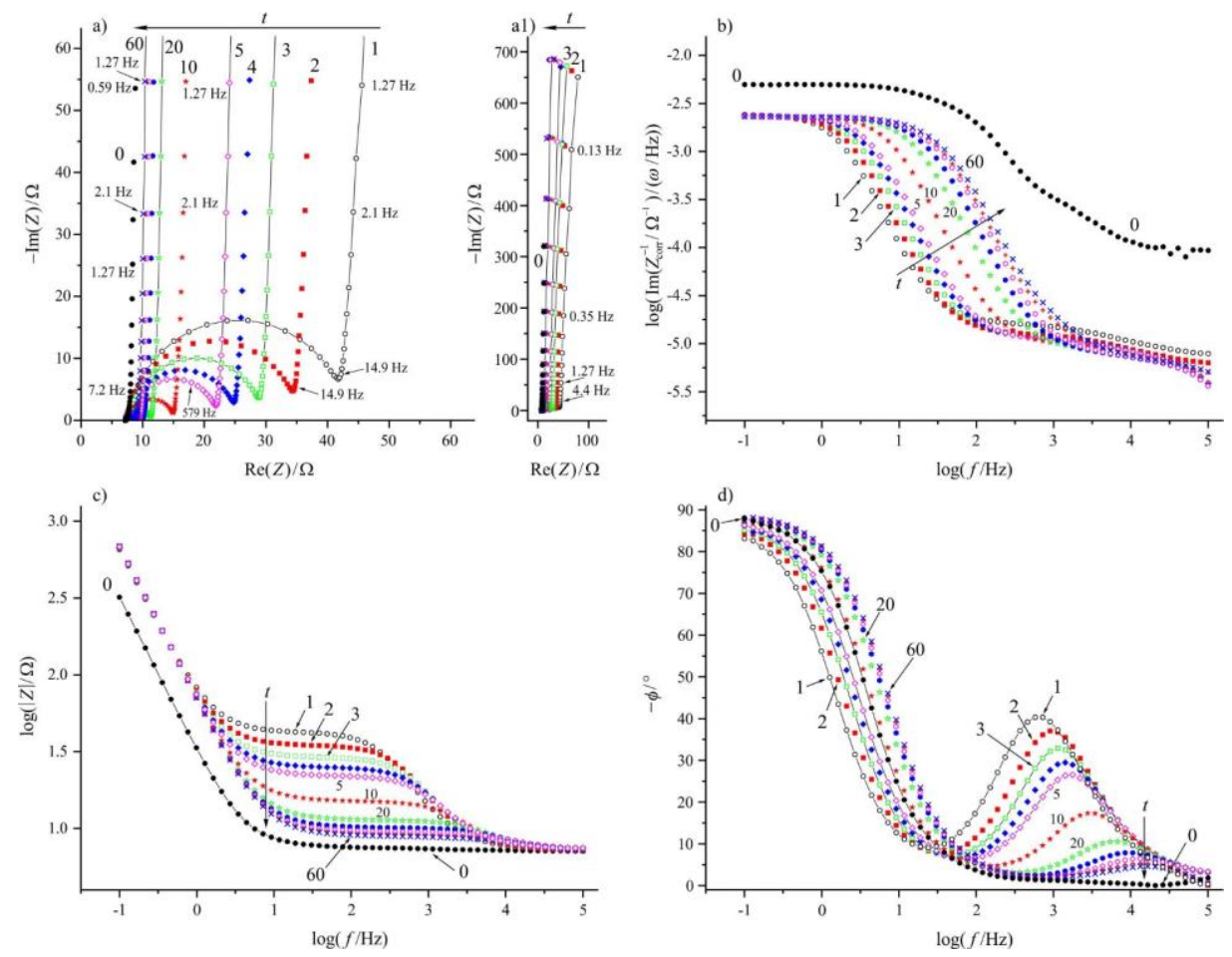

Fig. 3. a) Complex plane impedance plot for the "pristine" $\mathrm{Au}|\mathrm{PEDOT}| 0.1 \mathrm{M} \mathrm{H}_{2} \mathrm{SO}_{4}$ electrode at $E=0.4 \mathrm{~V}$ vs. SSCE (0: $\bullet$ ) and successive complex plane impedance diagrams recorded at the same electrode potential after

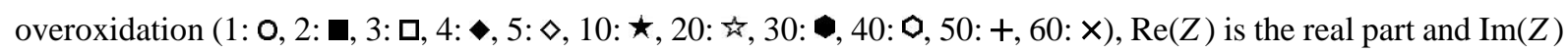
is the imaginary part of the complex impedance, respectively; a1) Low frequency part of the Argand diagrams in a); b) $\log (\operatorname{Im}(Y) / \omega)$ vs. $\log f$ plots (log(capacitance) vs. $\log f$ plots), where the admittance $Y$ is the reciprocal value of the impedance $\left(Z_{\text {corr }}\right)$ corrected for the uncompensated ohmic resistance, $f$ is the frequency, $\omega$ is the angular frequency of the perturbing signal.

c) magnitude ( $|\mathrm{Z}|$ ) vs. $\log f$ plots corresponding to a) and a1);

d) phase angle ( $-\phi$ ) vs. $\log f$ plots corresponding to a) and a1);

Starting times of the successive impedance measurements ("timestamps"): 1: 0 s, 2: $346.6 \mathrm{~s}, 3: 692.5 \mathrm{~s}, 4: 1039.4 \mathrm{~s}$, 5: 1386.7 s, 10: 3173.1 s, 20: 6713.9 s, 30: 10202.1 s, 40: 13766.4 s, 50: 17251.2 s, 60: 20777.4 s;

The solid lines are to guide the eye only: not curve fits.

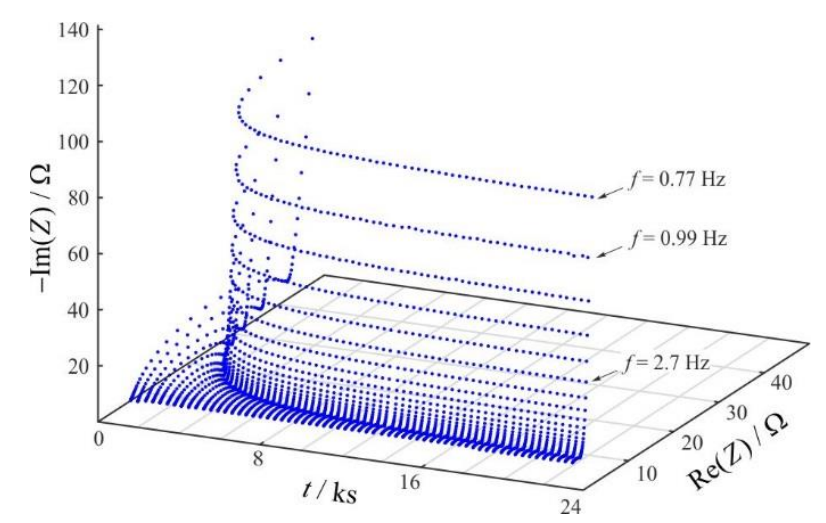

Fig. 4. Time dependence of the locus curves of recorded impedance spectra in the Argand-representation. $f$ is the frequency of the perturbing signal. 

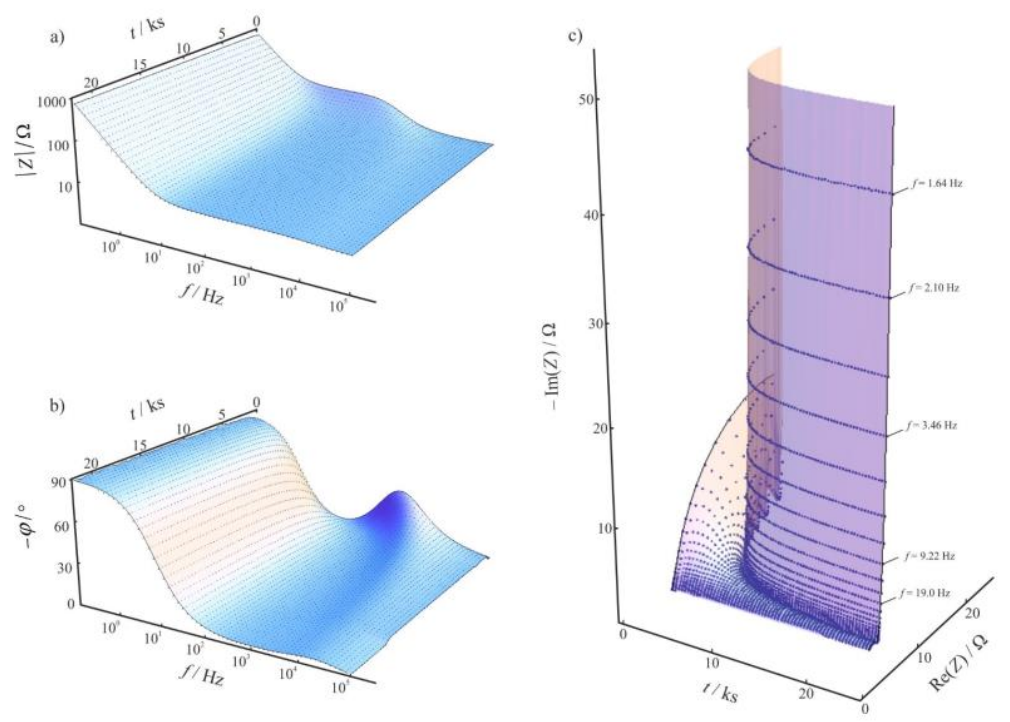

Fig.5. Results of a 3D complex interpolation. The absolute values (a) and phase angles (b) of measured impedances are shown as a function of time and frequency (discrete points). The continuous surface is created by a threedimensional bicubic spline interpolation algorithm implemented in LabVIEW [1]. In (c) both the measured data (discrete points) and the interpolated surface are transformed to a time-dependent complex plane representation. $f$ is the frequency of the perturbing signal.

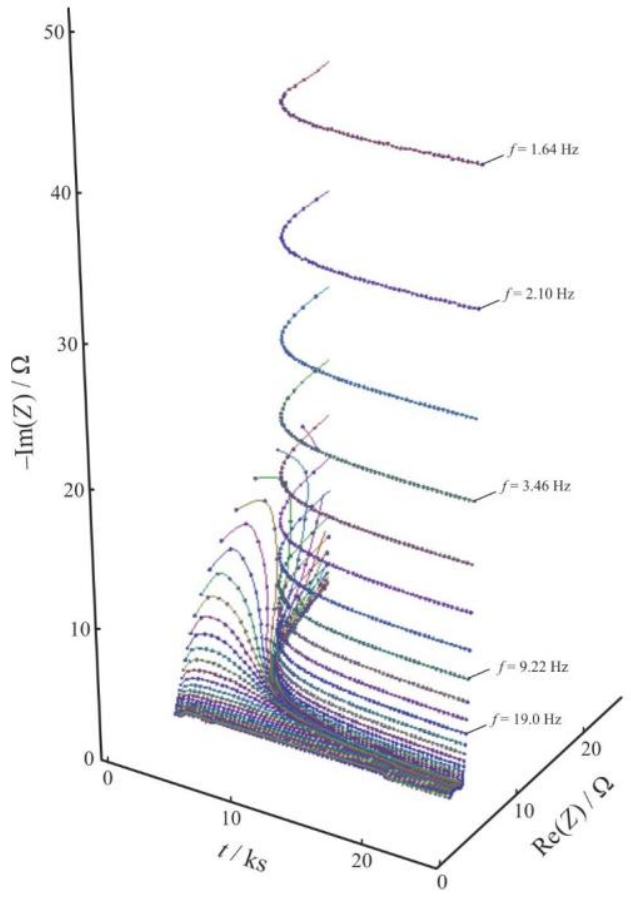

Fig.6. The measured impedance spectra and some isofrequential space curves created by spline interpolation shown in a three-dimensional (time dependent) complex plane representation. $f$ is the frequency of the perturbing signal. 

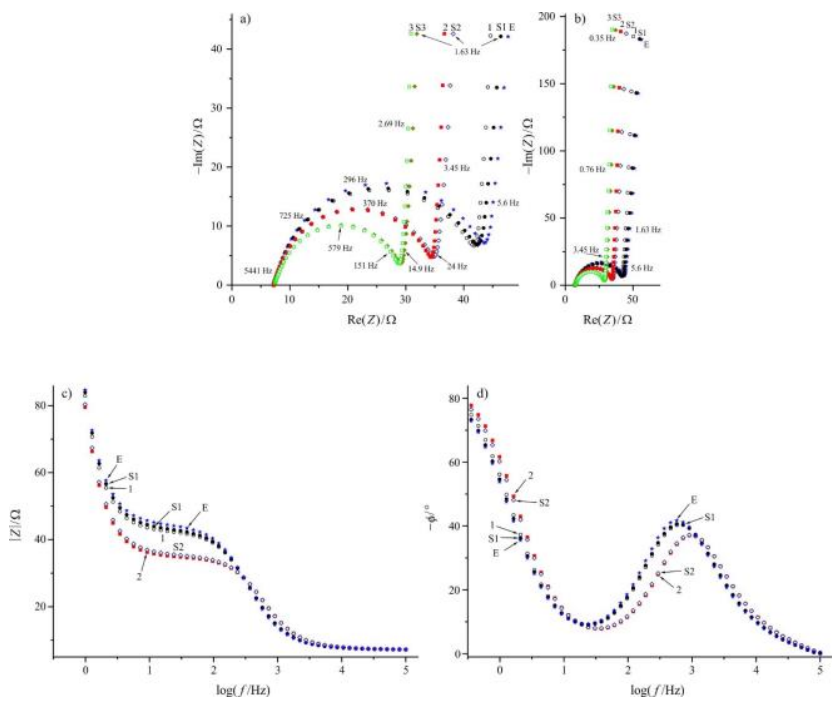

Fig.7. Directly measured and calculated (,instantaneous”) impedance spectra.

a) 1(0): experimental ("original") data points measured in the "frequency by frequency" mode of operation, i.e.

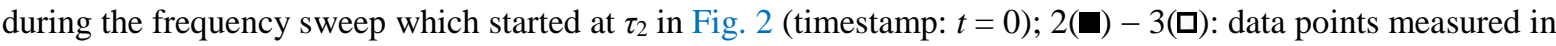
the "frequency by frequency" mode during the frequency sweeps that started at $t=346.6 \mathrm{~s}$ and $t=692.5 \mathrm{~s}$.

$\mathrm{S} 1(\bullet), \mathrm{S} 2(\diamond), \mathrm{S} 3(\bullet)$ : “Instantaneous" spectra calculated for $t=0, t=346.6 \mathrm{~s}$ and $t=692.5 \mathrm{~s}$, respectively.

$\mathrm{E}(\star)$ : calculated spectrum corresponding to the presumed end of the overoxidation process, $t=-53.0 \mathrm{~s}$

(corresponding to $\tau_{1}$ in Fig. 2). b) Low frequency part of the Argand diagrams presented in a);

c) magnitude ( $|\mathrm{Z}|)$ vs. $\log f$ plots corresponding to a) and b); d) phase angle $(-\phi)$ vs. $\log f$ plots corresponding to a) and $b) ; f$ is the frequency of the perturbing signal.
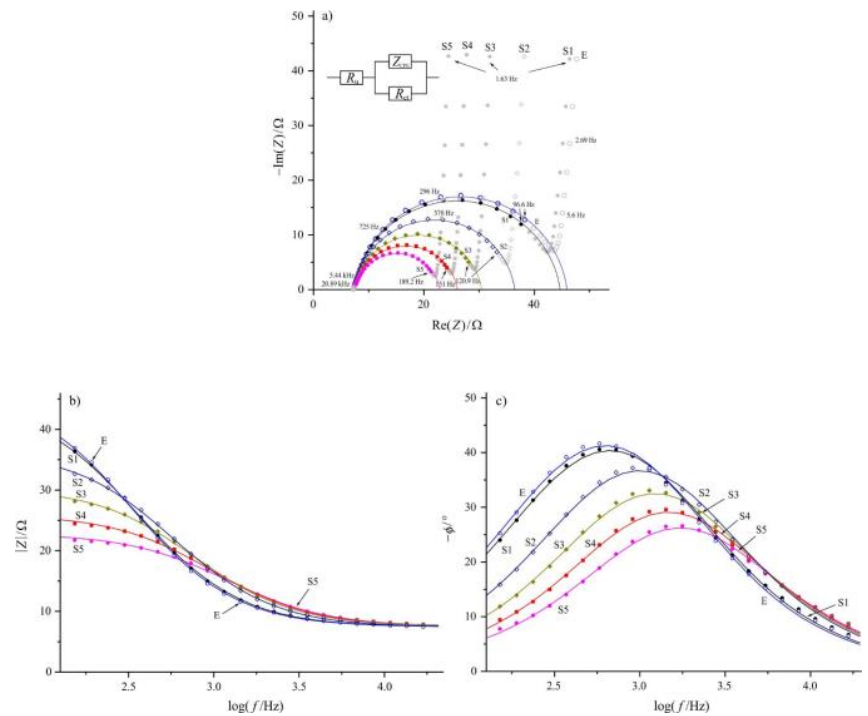

Fig.8. a) Complex plane impedance diagrams containing the ,instantaneous impedances”, calculated for $t=-53.0 \mathrm{~s}(\mathrm{E}: 0), t=0 \mathrm{~s}(\mathrm{~S} 1: \bullet), t=346.6 \mathrm{~s}(\mathrm{~S} 2: \diamond), t=692.5 \mathrm{~s}(\mathrm{~S} 3: \diamond), t=1039.4 \mathrm{~s}(\mathrm{~S} 4: \mathbf{\square})$, and $t=1386.7 \mathrm{~s}(\mathrm{~S} 5:$

), respectively. The solid curves were calculated (simulated) by using the "best-fit" parameters given in Table 1. Insert: the equivalent circuit analog used for CNLS fitting; b) magnitude ( $|\mathrm{Z}|$ ) vs. $\log f$ plots corresponding to a); c) phase angle $(-\phi)$ vs. $\log f$ plots corresponding to a); $f$ is the frequency of the perturbing signal; 


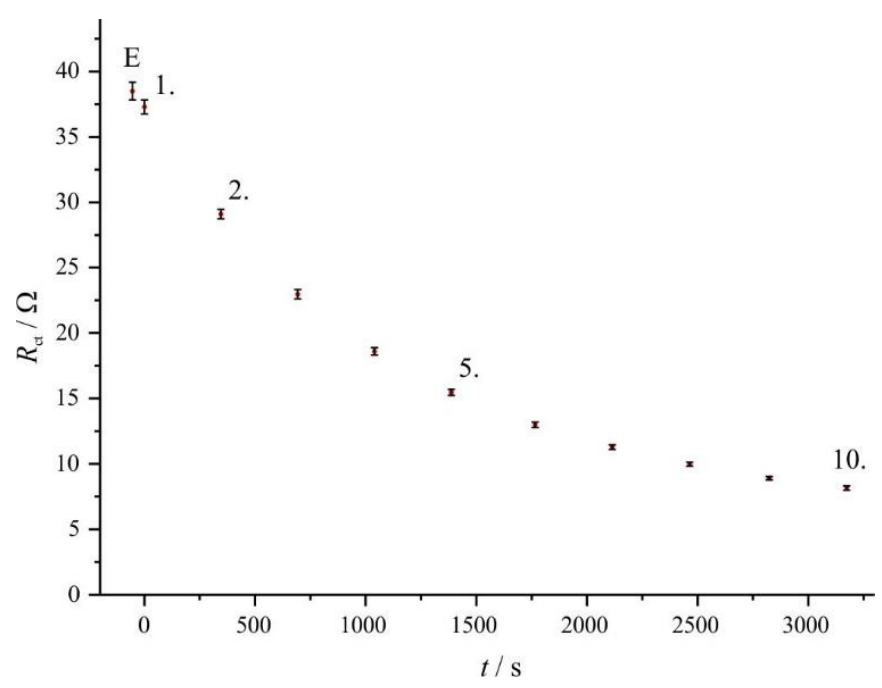

Fig.9. Time evolution of the charge transfer resistance during the experiment. The $R_{\mathrm{ct}}$ values are determined from the calculated impedance spectra (see Table 1). E is the $R_{\mathrm{ct}}$ value corresponding to the time instant ( $t=-53.0 \mathrm{~s}$ ) just after overoxidation of the film. 


\section{References}

[1] Z.B. Stoynov, B.S. Savova-Stoynov, Impedance study of non-stationary systems: four-dimensional analysis, J. Electroanal. Chem. 183 (1985) 133.

[2] Z. Stoynov, B. Savova, Instrumental error in impedance measurements of non-steady-state systems, J. Electroanal. Chem. 112 (1980) 157.

[3] B. Savova-Stoynov, Z. Stoynov, Four-dimensional estimation of the instantaneous impedance, Electrochim. Acta 37 (1992) 2353.

[4] K. Darowicki, Theoretical description of the measuring method of instantaneous impedance spectra, J. Electroanal. Chem. 486 (2000) 101.

[5] T. Breugelmans, J. Lataire, Th. Muselle, E. Tourwé, R. Pintelon, A. Hubin, Odd random phase multisine electrochemical impedance spectroscopy to quantify a non-stationary behaviour: Theory and validation by calculating an instantaneous impedance value, Electrochim. Acta 76 (2012) 375.

[6] Z. Stoynov, Nonstationary impedance spectroscopy, Electrochim. Acta 38 (1993) 1919.

[7] M. Keddam, Chr. Rakotomavo, H. Takenouti, Impedance of a porous electrode with an axial gradient of concentration, J. Appl. Electrochem. 14 (1984) 437.

[8] Z. Stoynov, Rotating Fourier transform-new mathematical basis for non-stationary impedance analysis, Electrochim. Acta. 37 (1992) 2357.

[9] G.G. Láng, M. Ujvári, S. Vesztergom, V. Kondratiev, J. Gubicza, K.J. Szekeres, The electrochemical degradation of poly(3,4-ethylenedioxythiophene) films electrodeposited from aqueous solutions, Z. Phys. Chem. 230 (2016) 1281.

[10] A. Zykwinska, W. Domagala, B. Pilawa, M. Lapkowski, Electrochemical overoxidation of poly(3,4ethylenedioxythiophene) - PEDOT studied by means of in situ ESR spectroelectrochemistry, Electrochim. Acta 50 (2005) 1625.

[11] M. Ujvári, M. Takács, S. Vesztergom, F. Bazsó, F. Ujhelyi, G.G. Láng, Monitoring of the electrochemical degradation of PEDOT films on gold using the bending beam method, J. Solid. State. Electrochem. 15 (2011) 2341.

[12] G.G. Láng, M. Ujvári, F. Bazsó, S. Vesztergom, F. Ujhelyi, In situ monitoring of the electrochemical degradation of polymer films on metals using the bending beam method and impedance spectroscopy, Electrochim. Acta 73 (2012) 59.

[13] M. Ujvári, J. Gubicza, V. Kondratiev, K.J. Szekeres, G.G. Láng, Morphological changes in electrochemically deposited poly(3,4-ethylenedioxythiophene) films during overoxidation, J. Solid. State. Electrochem. 19 (2015) 1247.

[14] M. Ujvári, G.G. Láng, S. Vesztergom, K.J. Szekeres, N. Kovács, J. Gubicza, Structural changes during the overoxidation of electrochemically deposited poly(3,4-ethylenedioxythiophene) films, J. Electrochem. Sci. Eng. 6 (2015) 77.

[15] G.G. Láng, C. Barbero, Laser techniques for the study of electrode processes, in: Monographs in electrochemistry, F. Scholz (Ed.), Springer, Berlin Heidelberg, 2012. 
[16] N. Kovács, M. Ujvári, G.G. Láng, P. Broekmann, S. Vesztergom, Characterization of the capacitance of a rotating ring-disk electrode, Instrum. Sci. Technol. 43 (2015) 633.

[17] J. Li, X-Q. Lin, Simultaneous determination of dopamine and serotonin on gold nanocluster/overoxidizedpolypyrrole composite modified glassy carbon electrode, Sens. Actuators B 124 (2007) 486.

[18] D.C. Martin, J. Wu, C.M. Shaw, Z. King, S.A. Spanninga, S. Richardson-Burns, J. Hendricks, J. Yang, The morphology of poly(3,4-ethylenedioxythiophene), Polym. Rev. 50 (2010) 340.

[19] Z. Zhuang, J. Li, R. Xu, D. Xiao, Electrochemical detection of dopamine in the presence of ascorbic acid using overoxidized polypyrrole/graphene modified electrodes, Int. J. Electrochem. Sci. 6 (2011) 2149.

[20] M. Irimia-Vladu, "Green" electronics: biodegradable and biocompatible materials and devices for sustainable future, Chem. Soc. Rev. 43 (2014) 588.

[21] G.G. Láng, M. Ujvári, Z. Dankházi, S. Vesztergom, K.J. Szekeres: Analysis of impedance spectra of electrochemically deposited PEDOT films recorded before and after overoxidation, 10th International Symposium on Electrochemical Impedance Spectroscopy, A Toxa, Spain, June 19 - 24, 2016, Book of Abstracts, p.16 (0720).

[22] J. Bobacka, A. Lewenstam, A. Ivaska, Electrochemical impedance spectroscopy of oxidized poly(3,4ethylenedioxythiophene) film electrodes in aqueous solutions, J. Electroanal. Chem. 489 (2000) 17.

[23] A. Stoyanova, V. Tsakova, Copper-modified poly(3,4-ethylenedioxythiophene) layers for selective determination of dopamine in the presence of ascorbic acid: I. Role of the polymer layer thickness, J. Solid State Electrochem. 14 (2010) 1947.

[24] W. Poppendieck, K.P. Hoffmann, in: J. Vander Sloten, P. Verdonck, M. Nyssen, J. Haueisen (Eds.), ECIFMBE 2008, IFCMBE Proceedings 22, Springer-Verlag, Berlin, Heidelberg, 2009, p. 2409.

[25] W.H. Press, S.A. Teukolsky, W.T. Vetterling, B.P. Flannery (Eds) Numerical Recipes in C++: The Art of Scientific Computing. 2nd ed. Cambridge: Cambridge University Press, 2002.

[26] G. Inzelt, G. Láng, Impedance analysis of poly(tetracyanoquinodimethane) electrodes: effect of electrolyte concentration and temperature, Electrochim. Acta 36 (1991) 1355.

[27] G. Inzelt, G. G. Láng, Electrochemical Impedance Spectroscopy (EIS) for Polymer Characterization, Ch. 3, in: Electropolymerization: Concepts, Materials and Applications, S. Cosnier, A. Karyakin (Eds.), WileyVCH Verlag GmbH \& Co. KGaA,Weinheim, 2010.

[28] G. Láng, K.E. Heusler, Comments on the ideal polarisability of electrodes displaying cpe-type capacitance dispersion, J. Electroanal. Chem. 481 (2000) 227.

[29] W.J. Albery, A.R. Mount, Transmission Lines for Conducting Polymers, Ch.4, in Electroactive Polymer Electrochemistry: Part 1: Fundamentals, M.E.G. Lyons (Ed.), Springer Science, New York, 1994.

[30] G. Láng, G. Inzelt, Some problems connected with impedance analysis of polymer film electrodes: effect of the film thickness and the thickness distribution, Electrochim Acta 36 (1991) 847.

[31] C. Gabrielli, H. Takenouti, O. Haas, A. Tsukada, Impedance investigation of the charge transport in filmmodified electrodes, J. Electroanal. Chem. 302 (1991) 59.

[32] Z. Stoynov, in Materials for Lithium-Ion Batteries 1999, C. Julien and Z. Stoynov, Eds, 85, p. 359, NATO Science Series, Kluwer Academic Publishers, Dordrecht, Boston, London, 2000.

[33] Z. Stoynov, B. Savova-Stoynov, T. Kossev, Non-stationary impedance analysis of lead/acid batteries, J. Power Sources 30 (1990) 275. 
[34] B. Savova-Stoynov, Z. Stoynov, Instantaneous Impedance Analysis, Key Eng. Mater. 59-60 (1991) 273.

[35] G. Láng, G. Inzelt, An advanced model of the impedance of polymer film electrodes, Electrochim Acta 44 (1999) 2037

[36] G. Inzelt, G. Láng, Model dependence and reliability of the electrochemical quantities derived from the measured impedance spectra of polymer modified electrodes, J. Electroanal. Chem. 378 (1994) 39.

[37] J. Bisquert, G. Garcia-Belmonte, F. Fabregat-Santiago, P. R. Bueno, Theoretical models for ac impedance of finite diffusion layers exhibiting low frequency dispersion, J. Electroanal. Chem. 475 (1999) 152.

[38] J. Tanguy, P. Vieil, G. Deniau, G. Lecayon, Study of the grafting and of the electrochemical polymerization of acrylic monomers on a metallic surface by impedance spectroscopy, Electrochim. Acta 38 (1993) 1501.

[39] M. Waller, R. G. Compton, Simultaneous alternating current impedance/electron spin resonance study of electrochemical doping in polypyrrole, J. Chem. Soc. Faraday Trans. 85 (1989) 977.

[40] G. Láng, J. Bácskai, G. Inzelt, Impedance analysis of polymer film electrodes, Electrochim. Acta 38 (1993) 773.

[41] K. Levenbeg, A method for the solution of certain nonlinear problems in least squares, Quart. Appl. Math., 2 (1944) 164.

[42] D.W. Marquardt, An algorithm for least-squares estimation of nonlinear parameters, SIAM J. Appl. Math. 11 (1963) 431.

[43] P. Valkó, S. Vajda, Advanced scientific computing in BASIC with applications in chemistry, biology and pharmacology, Data handling in science and technology, Vol. 4, Elsevier, Amsterdam, 1989.

[44] M.A. Komkova, E.V. Karpova, G.A. Sukhorukov, A.A. Sadovnikov, A.A. Karyakin, Estimation of continuity of electroactive inorganic films based on apparent anti-Ohmic trend in their charge transfer resistance, Electrochim. Acta 219 (2016) 588.

[45] G. Láng, K.E. Heusler, Remarks on the energetics of interfaces exhibiting constant phase element behaviour, J. Electroanal. Chem. 457 (1998) 257.

[46] G. Láng, K.E. Heusler, Comment on the ideal polarisability of electrodes displaying cpe-type capacitance dispersion, J. Electroanal. Chem. 481 (2000) 217. 